\title{
Dynamic complexity and bifurcation analysis of a host-parasitoid model with Allee effect and Holling type III functional response
}

\author{
Hua Liu' ${ }^{1}$, Kai Zhang ${ }^{1}$, Yong Ye ${ }^{1}$, Yumei Wei ${ }^{2^{*}}$ and Ming Ma
}

"Correspondence:

649118046@qq.com

2Experimental Teaching

Department, Northwest Minzu

University, Lanzhou, P.R. China

Full list of author information is

available at the end of the article

\section{Springer}

\begin{abstract}
In this paper, we focus on dynamics in a basic discrete-time system of host-parasitoid interaction. We perform local stability analysis of this system. Furthermore, both flip and Neimark-Sacker bifurcations are also analyzed in the interior of $R_{+}^{2}$ by using center manifold theorem and bifurcation theory. Finally, numerical simulations are deployed to validate our results with theoretical analysis and to exhibit the dynamical behaviors.
\end{abstract}

Keywords: Host-parasitoid system; Bifurcation; Center manifold theorem; Chaos control

\section{Introduction}

In the study of population dynamics, two kinds of mathematical systems are mainly used, namely the continuous-time system and the discrete-time system, of which the latter is more appropriate in the condition that population size is small or that population does not overlap. For example, many species of insects do not overlap in their offsprings, thus their populations are characterized by discrete-time systems [1].

In 1932, an American zoologist Allee proposed the Allee effect: group living is beneficial to the growth and survival of population, but if the population is too sparse or crowded, it will hinder its growth, as each species has its most suitable density to grow. The Allee effect mainly has two types. If the average growth rate of population at low density is negative, it is called the strong Allee effect; whereas the weak Allee effect means that the average growth rate of population is positive at zero density. The strong Allee effect proposes a population threshold, and that population density only exceeds this threshold to survive. In contrast, populations with weak Allee effects do not have this threshold. Recently, researchers have focused on the Allee effect on different ecosystems, including discrete-time systems [2-7] and continuous-time systems [8-14]. Zhao and Lv [15] study the dynamic complexity of a host-parasitoid system with a lower bound for host, and the form of Allee effect is $\frac{H(t)-n}{H(t)+m}$, where $m$ is an Allee effect constant, $n$ is the lower bound for the host.

The mutual restriction between species is a key point of population dynamics research, and this mutual restriction relationship can be represented by a function called functional response function, which can be divided into many classes according to different populations, such as Holling type and Beddington type. Veijo K. et al. [16] study the complex dynamics occurring in a basic discrete-time model of host-parasitoid interaction. Tang and

(c) The Author(s) 2019. This article is distributed under the terms of the Creative Commons Attribution 4.0 International License (http://creativecommons.org/licenses/by/4.0/), which permits unrestricted use, distribution, and reproduction in any medium, provided you give appropriate credit to the original author(s) and the source, provide a link to the Creative Commons license, and indicate if changes were made. 
Chen [17] report the dynamic complexities of host-parasitoid interactions with a Holling type II functional response, this discrete-time system is as follows:

$$
\left\{\begin{array}{l}
H(t+1)=H(t) \exp \left[r\left(1-\frac{H(t)}{K}\right)-\frac{a T P(t)}{1+a T_{n} H(t)}\right] \\
P(t+1)=H(t)\left[1-\exp \left(\frac{a T P(t)}{1+a T_{n} H(t)}\right)\right]
\end{array}\right.
$$

where $H(t)$ is the host population size in generation $t, P(t)$ is the parasitoid population size in generation $t, r$ is the intrinsic growth rate, $K$ is the carrying capacity of the environment, $T$ is the total time initially available, when hosts are exposed to parasitoids, $T_{n}$ is the handling time, which is between the host being encountered and search being resumed, $a$ is the instantaneous search rate. These parameters $T_{n}, T, a, K$, and $r$ are all positive constants.

Some ecological systems, although simple in mathematical expressions, have been designed to study the population temporal dynamics. In particular, the pioneering work in this field was initiated by May [18]. The significance of May's seminal work is inducting a new research area dealing with the complexities in the population dynamic systems. Tang and Chen study the complex dynamics of host-parasites with Holling type III functional response [17]. Oaten and Murdoch et al. discuss many factors that influence the stability of a continuous predator-prey system, especially the influence of Holling type III functional response $[19,20]$. In this paper, the host-parasitoid system with Allee effect and Holling type III functional response that we investigate is as follows:

$$
\left\{\begin{array}{l}
H(t+1)=H(t) \exp \left[\frac{r\left(1-\frac{H(t)}{K}\right)(H(t)-n)}{H(t)+m}-\frac{b T H(t) P(t)}{1+c H(t)+b T_{n} H(t)^{2}}\right] \\
P(t+1)=H(t)\left[1-\exp \left(-\frac{b T H(t) P(t)}{1+c H(t)+b T_{n} H(t)^{2}}\right)\right]
\end{array}\right.
$$

where $b, c$ are the parameters related to the Holling type III functional response $\frac{b T H(t) P(t)}{1+c H(t)+b T_{n} H(t)^{2}}$, and $b>0$ is a conversion factor, $c=a T_{n}$.

The outline of this paper is as follows. In Sect. 2, we perform the persistence analysis of system (1.2). In Sect. 3, we discuss the local stability of equilibrium points. We prove that under certain parametric conditions system (1.2) admits a bifurcation in Sect. 4. In Sect. 5, we verify our analytical results by numerical simulations. We conclude this study in the final section.

\section{Persistence analysis of the system}

Persistence analysis of a host-parasitoid system has great importance in understanding its biological relevance, and the persistence of system (1.2) in this paper is shown as follows.

Definition 2.1 ([21]) There exist positive constants $M_{1}, M_{2}$, which are independent of the solutions of the system, such that for any positive solution $(x(t), y(t))^{T}$ of the system, one has $M_{1} \leq \liminf _{t \rightarrow \infty} x(t) \leq \limsup _{t \rightarrow \infty} x(t) \leq M_{2}, M_{1} \leq \liminf _{t \rightarrow \infty} y(t) \leq$ $\lim \sup _{t \rightarrow \infty} y(t) \leq M_{2}, t=1,2, \ldots$, the system is permanent.

Lemma 2.1 ([22]) Assume that $\{x(t)\}$ satisfies $x(t)>0$ and $x(t+1) \leq x(t) \exp \{a-b x(t)\}$ for $t \in N$, where $a$ and $b$ are positive constants. Then $\lim \sup _{t \rightarrow \infty} x(t) \leq \frac{1}{b} \exp (a-1)$. 
Lemma 2.2 ([22]) Assume that $\{x(t)\}$ satisfies $x(t+1) \geq x(t) \exp \{a-b x(t)\}, t \geq t_{0}$, $\lim \sup _{t \rightarrow \infty} x(t) \leq x^{*}$, and $x\left(t_{0}\right)>0, t_{0} \in N$, where $a$ and $b$ are positive constants. Then $\liminf _{t \rightarrow \infty} x(t) \geq \min \left\{\frac{a}{b} \exp \left(a-b x^{*}\right), \frac{a}{b}\right\}$.

Theorem 2.1 For any solution $\{H(t), P(t)\}^{T}$ of system (1.2), we have lim $\sup _{t \rightarrow \infty} H(t) \leq M_{2}$, $\lim \sup _{t \rightarrow \infty} P(t) \leq M_{2}$, where $M_{2}=\frac{K \exp \left(\frac{r m}{K}+\frac{r n}{K}+r-1\right)}{r}$.

Proof For any $t \geq 0$, there exist $H(t)>0, P(t)>0$, and $r>0, n>0, K>0, m>0, T>0$, $T_{n}>0, c>0, b>0$. For the first equation of system (1.2), it follows that

$$
\begin{aligned}
H(t+1) & \\
& =H(t) \exp \left[\frac{r\left(1-\frac{H(t)}{K}\right)(H(t)-n)}{H(t)+m}-\frac{b T H(t) P(t)}{1+c H(t)+b T_{n} H^{2}(t)}\right] \\
& \leq H(t) \exp \left[\frac{r\left(1-\frac{H(t)}{K}\right)(H(t)-n)}{H(t)+m}\right] \\
& \leq H(t) \exp \left[\frac{-\frac{r}{K} H(t)(H(t)+m)+\left(\frac{r m}{K}+\frac{r n}{K}+r\right)(H(t)+m)-\left(\frac{r m^{2}}{K}+\frac{r n m}{K}+r m+r n\right)}{H(t)+m}\right] \\
& \leq H(t) \exp \left[\frac{r m}{K}+\frac{r n}{K}+r-\frac{r}{K} H(t)\right] .
\end{aligned}
$$

By using Lemma 2.1, one could easily obtain that

$$
\lim _{t \rightarrow \infty} H(t) \leq \frac{K \exp \left(\frac{r m}{K}+\frac{r n}{K}+r-1\right)}{r}=M_{2}
$$

For the second equation of system (1.2), it follows that

$$
\begin{aligned}
P(t+1) & =H(t)\left[1-\exp \left(-\frac{b T H(t) P(t)}{1+c H(t)+b T_{n} H^{2}(t)}\right)\right] \leq H(t) \\
& \leq H(t-1) \exp \left[\left(\frac{r m}{K}+\frac{r n}{K}+r\right)-\left(\frac{r}{K}\right) H(t-1)\right],
\end{aligned}
$$

then

$$
\limsup _{t \rightarrow \infty} P(t) \leq \frac{K \exp \left(\frac{r m}{K}+\frac{r n}{K}+r-1\right)}{r}=M_{2} .
$$

The proof is completed.

Theorem 2.2 For any solution $\{H(t), P(t)\}^{T}$ of system (1.2), assume that $\left(\frac{r m^{2}}{K}-r m-r n-\right.$ $\left.\frac{r m n}{K}\right)>0$ and $r-\frac{r\left(M_{2}+m\right)}{K}+\frac{r}{K} n>0$, we have $\liminf _{t \rightarrow \infty} H(t) \geq M^{\wedge} \geq M_{1}, \liminf _{t \rightarrow \infty} P(t) \geq$ $M^{*} \geq M_{1}$, where $M^{\wedge}=(-m+n+K) \exp \left(\frac{r n}{K}-\frac{r m}{K}+r-\frac{r}{K} M_{2}\right) \exp \left(-\frac{b T M_{2}}{2 \sqrt{b T_{n}}+c}\right)$, $M^{*}=\sqrt{\frac{\left\{1+\exp \left[r-\frac{r\left(M_{2}+m\right)}{K}+\frac{r}{K} n\right]\right\}\left[r-\frac{r\left(M_{2}+m\right)}{K}+\frac{r}{K} n\right]}{b T}}, M_{1}=\min \left\{M^{\wedge}, M^{*}\right\}$.

Proof From Theorem 2.1, we get $\lim \sup _{t \rightarrow \infty} H(t) \leq M_{2}, \limsup _{t \rightarrow \infty} P(t) \leq M_{2}$. For $\forall \varepsilon$, $\exists t_{1}>0$, and $t>t_{1}$, we have $H(t) \leq M_{2}+\varepsilon, P(t) \leq M_{2}+\varepsilon_{1}$. 
For the first equation of system (1.2) with $t>t_{1}$, it follows that

$$
\begin{aligned}
& H(t+1) \\
& =H(t) \exp \left[\frac{r\left(1-\frac{H(t)}{K}\right)(H(t)-n)}{H(t)+m}-\frac{b T H(t) P(t)}{1+c H(t)+b T_{n} H^{2}(t)}\right] \\
& \geq H(t) \exp \left[\frac{r(H(t)+m)-r m-\frac{r(H(t)+m)^{2}}{K}+\frac{2 r m}{K} H(t)+\frac{r}{K} m^{2}-r n+\frac{r}{K} n(H(t)+m)-\frac{r}{K} n m}{H(t)+m}\right] \\
& \quad \times \exp \left(-\frac{b T P(t)}{2 \sqrt{b T_{n}}+c}\right) .
\end{aligned}
$$

Assume that $\left(\frac{r m^{2}}{K}-r m-r n-\frac{r m n}{K}\right)>0$, we have

$$
\begin{aligned}
H(t+1) & \geq H(t) \exp \left[r-\frac{r(H(t)+m)}{K}+\frac{r}{K} n\right] \exp \left(-\frac{b T P(t)}{2 \sqrt{b T_{n}}+c}\right) \\
& \geq H(t) \exp \left[r-\frac{r(H(t)+m)}{K}+\frac{r}{K} n\right] \exp \left(-\frac{b T\left(M_{2}+\varepsilon_{1}\right)}{2 \sqrt{b T_{n}}+c}\right),
\end{aligned}
$$

by using Lemma 2.2, we get

$$
\begin{aligned}
& \lim \inf _{t \rightarrow \infty} H(t) \\
& \geq \frac{K}{r}\left(-\frac{r m}{K}+\frac{r n}{K}+r\right) \exp \left(\frac{r n}{K}-\frac{r m}{K}+r-\frac{r}{K}\left(M_{2}+\varepsilon_{1}\right)\right) \exp \left(-\frac{b T\left(M_{2}+\varepsilon_{1}\right)}{2 \sqrt{b T_{n}}+c}\right),
\end{aligned}
$$

and

$$
\lim \inf _{t \rightarrow \infty} H(t) \geq(n-m+K) \exp \left(\frac{r n}{K}-\frac{r m}{K}+r-\frac{r}{K} M_{2}\right) \exp \left(-\frac{b T M_{2}}{2 \sqrt{b T_{n}}+c}\right)=M^{\wedge}
$$

For the first equation and the second equation of system (1.2), we have

$$
\begin{aligned}
& P(t)=\sqrt{\frac{\ln Q(1+Q) M}{b T}}, \\
& \text { where } Q=\exp \left[\frac{r(1-H(t) / K)(H(t)-n)}{H(t)+m}\right], M=1+c H(t)+b T_{n} H(t)^{2},
\end{aligned}
$$

and

$$
\begin{aligned}
P(t) & =\sqrt{\frac{\left(1+c H(t)+b T_{n} H(t)^{2}\right)\left(1+\exp \left[\frac{r(1-H(t) / K)(H(t)-n)}{H(t)+m}\right]\right) \ln \exp \left[\frac{r(1-H(t) / K)(H(t)-n)}{H(t)+m}\right]}{b T}} \\
& \geq \sqrt{\frac{\left(1+\exp \left[\frac{r(1-H(t) / K)(H(t)-n)}{H(t)+m}\right]\right)\left[\frac{r(1-H(t) / K)(H(t)-n)}{H(t)+m}\right]}{b T}} \\
& \geq \sqrt{\frac{\left(1+\exp \left[r-\frac{r(H(t)+m)}{K}+\frac{r}{K} n\right]\right)\left[r-\frac{r(H(t)+m)}{K}+\frac{r}{K} n\right]}{b T} .}
\end{aligned}
$$


From Theorem 2.1, we have $H(t) \leq M_{2}$, then

$$
P(t) \geq \sqrt{\frac{\left\{1+\exp \left[r-\frac{r\left(M_{2}+m\right)}{K}+\frac{r}{K} n\right]\right\}\left[r-\frac{r\left(M_{2}+m\right)}{K}+\frac{r}{K} n\right]}{b T}} .
$$

Assume that $r-\frac{r\left(M_{2}+m\right)}{K}+\frac{r}{K} n>0$, let $\sqrt{\frac{\left\{1+\exp \left[r-\frac{r\left(M_{2}+m\right)}{K}+\frac{r}{K} n\right]\right\}\left[r-\frac{r\left(M_{2}+m\right)}{K}+\frac{r}{K} n\right]}{b T}}=M^{*}$, we have $P(t) \geq M^{*}$, then

$$
\lim \inf _{t \rightarrow \infty} P(t) \geq M^{*}
$$

The proof is completed.

Theorem 2.3 From Lemmas 2.1 and 2.2, if $\left(\frac{r m^{2}}{K}-r m-r n-\frac{r m n}{K}\right)>0$ and $r-\frac{r\left(M_{2}+m\right)}{K}+\frac{r}{K} n>$ 0 , where $M_{2}=\frac{K \exp \left(\frac{r m}{K}+\frac{r n}{K}+r-1\right)}{r}$, then system (1.2) is permanent.

\section{Stability of equilibria}

In this section, we determine the existence of equilibria of system (1.2) and then study their stability at each equilibrium point. Finally, conditions for the existence of a flip bifurcation and a Neimark-Sacker bifurcation are derived [23, 24].

For simplicity, system (1.2) can be rewritten as follows:

$$
\left\{\begin{array}{l}
x \rightarrow x \exp \left[\frac{r\left(1-\frac{x}{K}\right)(x-n)}{x+m}-\frac{b T x y}{1+c x+b T_{n} x^{2}}\right] \\
y \rightarrow x\left[1-\exp \left(-\frac{b T_{x}}{1+c x+b T_{n} x^{2}}\right)\right]
\end{array}\right.
$$

There are four non-negative equilibrium points for system (3.1). The total extinction solution whereby no species is able to survive is $E_{0}(0,0)$, and the boundary equilibrium point that only one species survives is $E_{1}(n, 0), E_{2}(K, 0)$, and the coexistence solution for the two species is $E_{*}\left(x_{*}, y_{*}\right)$, when $Q>1$, that is, $n<H(t)<K$.

$$
\left\{\begin{array}{l}
x_{*}=\frac{M \ln Q}{b T \sqrt{\frac{M(1+Q) \ln Q}{b T}}}, \\
y_{*}=\sqrt{\frac{M(1+Q) \ln Q}{b T}},
\end{array}\right.
$$

where $Q$ is the net rate of the increase in host per generation, and

$$
Q=\exp \left[\frac{r\left(1-\frac{x_{*}}{K}\right)\left(x_{*}-n\right)}{x_{*}+m}\right], \quad M=1+c x_{*}+b T_{n} x_{*}^{2} .
$$

(1) The Jacobian matrix of the system at $E_{0}(0,0)$ is

$$
J_{0}(0,0)=\left(\begin{array}{cc}
\exp \left(-\frac{r n}{m}\right) & 0 \\
0 & 0
\end{array}\right)
$$

and we can get eigenvalues

$$
\lambda_{1}=\exp \left(-\frac{r n}{m}\right), \quad \lambda_{2}=0,
$$

from this, it can be concluded that $E_{0}$ is a stable node $\left(\left|\lambda_{1}\right|<1\right)$. 
(2) The Jacobian matrix of the system at $E_{1}(n, 0)$ is

$$
J_{1}(n, 0)=\left(\begin{array}{cc}
1+r n\left(\frac{1-\frac{n}{K}}{m+n}\right) & -\frac{b T n^{2}}{1+c n+b T_{n} n^{2}} \\
0 & \frac{b T n^{2}}{1+c n+b T_{n} n^{2}}
\end{array}\right)
$$

if $b T n^{2}>1+c n+b T_{n} n^{2}$, then $E_{1}(n, 0)$ is a source $\left(\left|\lambda_{1}\right|>1,\left|\lambda_{2}\right|>1\right)$; if $b T n^{2}<1+c n+b T_{n} n^{2}$, then $E_{1}(n, 0)$ is a saddle $\left(\left|\lambda_{1}\right|>1,\left|\lambda_{2}\right|<1\right)$.

(3) The Jacobian matrix of system at $E_{2}(K, 0)$ is

$$
J_{2}(K, 0)=\left(\begin{array}{cc}
1-\frac{r(K-n)}{m+K} & -\frac{b T K^{2}}{1+c n+b T_{n} n^{2}} \\
0 & \frac{b T K^{2}}{1+c K+b T_{n} K^{2}}
\end{array}\right)
$$

if $b T K^{2}>1+c K+b T_{n} K^{2}$ and $r(K-n)>2(K+m)$, then $E_{2}(K, 0)$ is a source $\left(\left|\lambda_{1}\right|>1,\left|\lambda_{2}\right|>1\right)$; if $b T K^{2}>1+c K+b T_{n} K^{2}, r(K-n)<2(K+m)$, or $b T K^{2}<1+c K+b T_{n} K^{2}, r(K-n)>2(K+m)$, then $E_{2}(K, 0)$ is a saddle $\left(\left|\lambda_{1}\right|>1,\left|\lambda_{2}\right|<1\right)$; and if $b T K^{2}<1+c K+b T_{n} K^{2}, r(K-n)<2(K+m)$, then $E_{2}(K, 0)$ is a sink $\left(\left|\lambda_{1}\right|<1,\left|\lambda_{2}\right|<1\right)$.

(4) The Jacobian matrix of system at $E_{*}\left(x_{*}, y_{*}\right)$ is

$$
J_{*}\left(x_{*}, y_{*}\right)=\left(\begin{array}{cc}
1+r x_{*} G-H & -\frac{b T x_{*}^{2}}{M} \\
1-\exp \left(-\frac{b T x_{*} y_{*}}{M}\right)+H \exp \left(-\frac{b T x_{*} y_{*}}{M}\right) & L
\end{array}\right),
$$

where $G=\frac{\left(1-\frac{x_{*}}{K}\right)(m+n)}{\left(x_{*}+m\right)^{2}}-\frac{x_{*}-n}{\left(x_{*}+m\right) K}, H=\frac{b T x_{*} y_{*}-b^{2} T T_{n} x_{*}^{3} y_{*}}{M^{2}}, L=\frac{b T x_{*}^{2}}{M} \exp \left(-\frac{b T x_{*} y_{*}}{M}\right)$.

The characteristic equation of $J_{*}$ is given by

$$
F(\lambda)=\lambda^{2}+\operatorname{tra}\left(x_{*}, y_{*}\right) \lambda+\operatorname{det}\left(x_{*}, y_{*}\right)=0
$$

where

$$
\begin{aligned}
\operatorname{tra} J_{*} & =-\left[1+r x_{*} G-H+L\right], \\
\operatorname{det} J_{*} & =r x_{*} G L+\frac{b T x_{*}^{2}}{M},
\end{aligned}
$$

and

$$
\begin{aligned}
& F(1)=\frac{b T x_{*}^{2}}{M}+H-L-r x_{*} G+r x_{*} G L, \\
& F(-1)=\frac{b T x_{*}^{2}}{M}-H+L+r x_{*} G+r x_{*} G L+2 .
\end{aligned}
$$

In order to discuss the stability of $E_{*}$, we first give the following lemma [25].

Lemma 3.1 Let $F(\lambda)=\lambda^{2}+$ tra $\lambda+$ det. Assume that $F(1)>0$, and $\lambda_{1}, \lambda_{2}$ are the roots of $F(\lambda)=0$. Then

i. $\left|\lambda_{1}\right|<1,\left|\lambda_{2}\right|>1$ (or $\left|\lambda_{1}\right|>1$ and $\left.\left|\lambda_{2}\right|<1\right)$ if and only if $F(-1)<0$;

ii. $\left|\lambda_{1}\right|<1,\left|\lambda_{2}\right|<1$ if and only if $F(-1)>0$ and $\mathrm{det}<1$;

iii. $\left|\lambda_{1}\right|>1,\left|\lambda_{2}\right|>1$ if and only if $F(-1)>0$ and $\operatorname{det}>1$; 
iv. $\lambda_{1}=-1,\left|\lambda_{2}\right| \neq 1$ if and only if $F(-1)=0$ and tra $\neq 0,2$

$\mathrm{v} . \lambda_{1}, \lambda_{2}$ are complex and $\left|\lambda_{1}\right|=\left|\lambda_{2}\right|=1$ if and only if tra $^{2}-4 \operatorname{det}<0$, det $=1$.

Let $\lambda_{1}$ and $\lambda_{2}$ be two roots of $F(\lambda)$, which are eigenvalues of the fixed point $E_{*}$. The fixed point $E_{*}$ is a sink or locally asymptotically stable if $\left|\lambda_{1}\right|<1$ and $\left|\lambda_{2}\right|<1$. $E_{*}$ is called a source if $\left|\lambda_{1}\right|>1$ and $\left|\lambda_{2}\right|>1$. A source is locally unstable. $E_{*}$ is called a saddle if $\left|\lambda_{1}\right|>1$ and $\left|\lambda_{2}\right|<1$ (or $\left|\lambda_{1}\right|<1$ and $\left|\lambda_{2}\right|>1$ ). And $E_{*}$ is non-hyperbolic if either $\left|\lambda_{1}\right|=1$ or $\left|\lambda_{2}\right|=1$.

Now, the following theorems show the stability of a positive fixed point of system (3.1).

Theorem 3.1 System (3.1) has a positive fixed point $E_{*}$ and

(1) It is a saddle if the following condition holds: $r<\frac{H-L-2-\frac{b T x_{*}^{2}}{M}}{x_{*} G+x_{*} G L}$;

(2) It is a sink if the condition holds: $r>\frac{H-L-2-\frac{b T x_{*}^{2}}{M}}{x_{*} G+x_{*} G L}$ and $r<\frac{1-\frac{b T x_{*}^{2}}{M}}{x_{*} G L}$;

(3) It is a source if one of the following conditions holds: $r>\frac{H-L-2-\frac{b T x_{*}^{2}}{M}}{x_{*} G+x_{*} G L}$ and $r>\frac{1-\frac{b T x_{*}^{2}}{M}}{x_{*} G L}$;

(4) It is non-hyperbolic if the condition holds:
(a) $r=\frac{H-L-2-\frac{b T x_{*}^{2}}{M}}{x_{*} G+x_{*} G L}$ and $r \neq \frac{H-L-1}{x_{*} G}, r \neq \frac{H-L+1}{x_{*} G}$;
(b) $r=\frac{1-\frac{b T x_{*}^{2}}{M}}{x_{*} G L}$ and $\frac{H-L-3}{x_{*} G}<r<\frac{H-L+1}{x_{*} G}$.

We can easily see that one of the eigenvalues of fixed point $E_{*}$ is -1 and the other is neither 1 nor -1 if term (a) of Theorem 3.1 holds. If term (b) holds, then the eigenvalues of $E_{*}$ are a pair of complex conjugate eigenvalues with modulus one. In the following section, we study the flip bifurcation and the N-S bifurcation.

\section{Bifurcation}

In this section, we discuss the flip bifurcation and the N-S bifurcation of system (3.1), and we choose $r$ as a bifurcation parameter for studying bifurcations.

\subsection{Flip bifurcation}

From Theorem 3.1(4)(a), system (3.1) has a unique positive equilibrium $E_{*}$, the corresponding eigenvalues are $\lambda_{1}=-1, \lambda_{2}=2+r x_{*} G-H+L$ with $\left|\lambda_{2}\right| \neq 1$.

By selecting arbitrary parameters $\left(r_{1}, m, K, c, b, T, T_{n}, n\right)$, we write system (3.1) in the form

$$
\left\{\begin{array}{l}
x \rightarrow x \exp \left[\frac{r_{1}\left(1-\frac{x}{R}\right)(x-n)}{x+m}-\frac{b T x y}{1+c x+b T_{n} x^{2}}\right] \\
y \rightarrow x\left[1-\exp \left(-\frac{b T x y}{1+c x+b T_{n} x^{2}}\right)\right]
\end{array}\right.
$$

Let $u=x-x_{*}, v=y-y_{*}, \delta=r-r_{1}$, system (4.1) becomes

$$
\left\{\begin{aligned}
u \rightarrow & a_{100} u+a_{010} v+a_{001} \delta+a_{200} u^{2}+a_{110} u v+a_{101} u \delta+a_{011} v \delta \\
& +a_{020} v^{2}+a_{002} \delta^{2}+a_{300} u^{3} \\
& +a_{210} u^{2} v+a_{201} u^{2} \delta+a_{111} u v \delta+a_{102} u \delta^{2}+a_{120} u v^{2}+a_{021} v^{2} \delta \\
& +a_{012} v \delta^{2}+a_{003} \delta^{3}+a_{030} v^{3}+O(4), \\
v \rightarrow & b_{100} u+b_{010} v+b_{200} u^{2}+b_{110} u v+b_{300} u^{3}+b_{210} u^{2} v+b_{020} v^{2} \\
& +b_{120} u v^{2}+b_{030} v^{3}+O(4),
\end{aligned}\right.
$$


where

$$
\begin{aligned}
& a_{100}=1+r_{1} x_{*} G-H, \quad a_{010}=-\frac{b T x_{*}^{2}}{M}, \\
& a_{001}=\frac{x_{*}\left(1-\frac{x_{*}}{K}\right)\left(x_{*}-n\right)}{x_{*}+m}, \\
& a_{200}=\frac{r_{1} G}{2}+r_{1} x_{*}\left[(m+n)\left(\frac{\frac{x_{*}-m}{K}-2}{2\left(x_{*}+m\right)^{3}}-\frac{1}{2 K\left(x_{*}+m\right)^{2}}\right)\right] \\
& -\frac{b T y_{*}-3 b^{2} T T_{n} x_{*}^{2} y^{*}}{2 M^{2}}+\frac{\left(b T x^{*} y^{*}-b^{2} T T_{n} x_{*}^{3} y_{*}\right)\left(2 c+4 b T_{n} x_{*}\right)}{2 M^{3}} \\
& +\left(1+r_{1} x_{*} G-H\right)\left(\frac{r_{1} G}{2}-\frac{H}{2 x_{*}}\right), \\
& a_{110}=\frac{-b T x_{*}\left(1+r_{1} x_{*} G-H\right)}{2 M}-\frac{b T x_{*}-b^{2} T T_{n} x_{*}^{3}}{2 M^{2}}, \\
& a_{101}=\frac{x_{*} G}{2}+\frac{\left(1+r_{1} x_{*} G-H\right)\left(x_{*}-n\right)\left(1-\frac{x_{*}}{K}\right)}{2\left(x_{*}+m\right)}, \\
& a_{011}=-\frac{b T x_{*}^{2}\left(x_{*}-n\right)\left(1-\frac{x_{*}}{K}\right)}{2 M\left(x_{*}+m\right)}, \quad a_{002}=\frac{x_{*}\left(1-\frac{x_{*}}{K}\right)^{2}\left(x_{*}-n\right)^{2}}{2\left(x_{*}+m\right)^{2}}, \\
& a_{300}=r_{1}(m+n)\left[\frac{\frac{x_{*}-m}{K}-2}{3\left(x_{*}+m\right)^{3}}-\frac{1}{3 K\left(x_{*}+m\right)^{2}}+\frac{x_{*}}{K\left(x_{*}+m\right)^{3}}-\frac{x_{*}\left(\frac{x_{*}-m}{K}-2\right)}{\left(x_{*}+m\right)^{4}}\right] \\
& +\frac{b^{2} T T_{n} x_{*} y_{*}}{M^{2}} \\
& +\frac{2 b c T y_{*}+6 b^{2} T T_{n} x_{*} y_{*}-6 b^{2} c T T_{n} x_{*}^{2} y_{*}-14 b^{3} T T_{n} x_{*}^{3} y_{*}}{3 M^{3}} \\
& -\frac{\left(c+2 b T_{n} x_{*}\right)^{2}\left(b T x_{*} y_{*}-b^{2} T T_{n} x_{*}^{3} y_{*}\right)}{M^{4}} \\
& +\left(\frac{r_{1} G}{3}-\frac{H}{3 x}\right)\left\{r_{1} G+r x_{*}\left[(m+n)\left(\frac{\frac{x_{*}-m}{K}-2}{\left(x_{*}+m\right)^{3}}-\frac{1}{K\left(x_{*}+m\right)^{2}}\right)\right]\right. \\
& \left.-\frac{b T y_{*}-3 b^{2} T T_{n} x_{*}^{2} y^{*}}{M_{2}}\right\} \\
& +\left(r_{1} G-\frac{H}{x}\right)\left[\frac{\left(b T x^{*} y^{*}-b^{2} T T_{n} x_{*}^{3} y_{*}\right)\left(2 c+4 b T_{n} x_{*}\right)}{3 M^{3}}-\frac{a_{200}}{6}\right] \\
& +\left(1+r_{1} x_{*} G-H\right)\left[\frac{r_{1}}{6}\left[\frac{\frac{x_{*}-m}{K}-2}{\left(x_{*}+m\right)^{3}}-\frac{1}{K\left(x_{*}+m\right)^{2}}\right]\right. \\
& \left.+\frac{b^{2} T T_{n} x_{*} y_{*}}{3 M^{2}}+\frac{\left(b T y^{*}-b^{2} T T_{n} x_{*}^{2} y_{*}\right)\left(c+2 b T_{n} x_{*}\right)}{3 M^{3}}\right], \\
& a_{102}=\frac{\left(1-\frac{x_{*}}{K}\right)\left(x_{*}-n\right)}{x_{*}+m}\left[\frac{x_{*} G}{3}+\frac{\left(1+r_{1} x_{*} G-H\right)\left(x_{*}-n\right)\left(1-\frac{x_{*}}{K}\right)}{6(x+m)}\right], \quad a_{020}=\frac{b^{2} T^{2} x_{*}^{3}}{6 M^{2}}, \\
& a_{201}=\frac{G}{6}+x_{*}(m+n)\left(\frac{\frac{x_{*}-m}{K}-2}{6\left(x_{*}+m\right)^{3}}-\frac{1}{6 K\left(x_{*}+m\right)^{2}}\right) \\
& +\frac{\left(1-\frac{x_{*}}{K}\right)\left(x_{*}-n\right)}{6\left(x_{*}+m\right)}\left\{r_{1} G+r_{1} x_{*}\left[(m+n)\left(\frac{\frac{x_{*}-m}{K}-2}{\left(x_{*}+m\right)^{3}}-\frac{1}{K\left(x_{*}+m\right)^{2}}\right)\right]\right. \\
& \left.-\frac{b T y_{*}-3 b^{2} T^{2} x_{*}^{2} y^{*}}{6 M^{2}}-\frac{\left(b T x^{*} y^{*}-b^{2} T^{2} x_{*}^{3} y_{*}\right)\left(c+2 b T x_{*}\right)}{3 M^{3}}\right\}
\end{aligned}
$$




$$
\begin{aligned}
& +\left(\frac{r_{1} G}{6}-\frac{H}{3 x_{*}}\right)\left[x_{*} G+\frac{\left(1-\frac{x_{*}}{K}\right)\left(x_{*}-n\right)\left(1+r_{1} x_{*} G-H\right)}{x_{*}+m}\right]+\frac{1}{6} G\left(1+r_{1} x_{*} G-H\right), \\
& a_{111}=-\frac{\left(b T x_{*}-b^{2} T T_{n} x_{*}^{3}\right)\left(1-\frac{x_{*}}{K}\right)\left(x_{*}-n\right)}{6 M^{2}\left(x_{*}+m\right)} \\
& -\frac{b T x_{*}}{6 M}\left[x_{*} G+\frac{\left(1-\frac{x_{*}}{K}\right)\left(x_{*}-n\right)\left(1+r_{1} x_{*} G-H\right)}{x_{*}+m}\right] \text {, } \\
& a_{120}=\frac{b T x_{*}}{M}\left[\frac{b T x_{*}-b^{2} T T_{n} x_{*}^{3}}{3 M^{2}}+\frac{b T x_{*}\left(1+r_{1} x_{*} G-H\right)}{6 M}\right], \quad a_{030}=-\frac{b^{3} T^{3} x_{*}^{4}}{6 M^{3}}, \\
& a_{003}=\frac{x_{*}\left(1-\frac{x_{*}}{K}\right)^{3}\left(x_{*}-n\right)^{3}}{6\left(x_{*}+m\right)^{3}}, \quad b_{100}=1-\exp \left(-\frac{b T x_{*} y_{*}}{M}\right)+H \exp \left(-\frac{b T x_{*} y_{*}}{M}\right) \text {, } \\
& b_{010}=L, \quad b_{110}=\exp \left(-\frac{b T x_{*} y_{*}}{M}\right)\left[\frac{b T x_{*}-b^{2} T T_{n} x_{*}^{3}}{M^{2}}+\frac{b T x_{*}(1-H)}{M}\right] \text {, } \\
& b_{200}=\exp \left(-\frac{b T x_{*} y_{*}}{M}\right)\left[\frac{H-H^{2}}{2 x_{*}}+\frac{b T y_{*}-3 b^{2} T T_{n} x_{*}^{2} y^{*}}{2 M^{2}}\right. \\
& \left.-\frac{\left(b T x^{*} y^{*}-b^{2} T T_{n} x_{*}^{3} y_{*}\right)\left(c+2 b T_{n} x_{*}\right)}{M^{3}}\right] \text {, } \\
& b_{110}=\exp \left(-\frac{b T x_{*} y_{*}}{M}\right)\left[\frac{b T x_{*}-b^{2} T T_{n} x_{*}^{3}}{2 M^{2}}+\frac{b T x_{*}(1-H)}{2 M}\right] \text {, } \\
& b_{020}=-\frac{b^{2} T^{2} x_{*}^{3}}{2 M^{2}} \exp \left(-\frac{b T x_{*} y_{*}}{M}\right) \text {, } \\
& b_{120}=\frac{b T x_{*}}{2 M} \exp \left(-\frac{b T x_{*} y_{*}}{M}\right)\left[-\frac{H}{y_{*}}-\frac{b T x_{*}}{M}(1-H)\right] \text {, } \\
& b_{300}=\exp \left(-\frac{b T x_{*} y_{*}}{M}\right)(1-H) \\
& \times\left(-\frac{H^{2}}{6 x_{*}^{2}}-\frac{b^{2} T T_{n} x_{*} y_{*}}{3 M^{2}}-\frac{\left(b T x^{*} y^{*}-b^{2} T T_{n} x_{*}^{2} y_{*}\right)\left(c+2 b T_{n} x_{*}\right)}{3 M^{3}}\right) \\
& +\frac{H}{x_{*}} \exp \left(-\frac{b T x_{*} y_{*}}{M}\right)(1-H) \\
& \times\left(\frac{\left(b T x^{*} y^{*}-b^{2} T T_{n} x_{*}^{3} y_{*}\right)\left(c+2 b T_{n} x_{*}\right)}{3 M^{3}}-\frac{b T y_{*}-3 b^{2} T T_{n} x_{*}^{2} y^{*}}{6 M^{2}}\right), \\
& b_{210}=\exp \left(-\frac{b T x_{*} y_{*}}{M}\right)\left(1-\frac{b T x_{*} y_{*}}{M}\right) \\
& \times\left(\frac{b T-3 b^{2} T T_{n} x_{*}^{2}}{6 M^{2}}-\frac{\left(b T x^{*}-b^{2} T T_{n} x_{*}^{3}\right)\left(c+2 b T_{n} x_{*}\right)}{3 M^{3}}\right) \\
& -\frac{1}{6} \exp \left(-\frac{b T x_{*} y_{*}}{M}\right)\left(\frac{b T H}{M}(1-H)-\frac{H^{2}}{x_{*} y_{*}}+\frac{H(1-H)}{x_{*} y_{*}}\right), \\
& b_{030}=\frac{b^{3} T^{3} x_{*}^{4}}{6 M^{3}} \exp \left(-\frac{b T x_{*} y_{*}}{M}\right) \text {. }
\end{aligned}
$$

We construct an invertible matrix:

$$
T=\left(\begin{array}{cc}
a_{010} & a_{010} \\
-1-a_{100} & \lambda_{2}-a_{100}
\end{array}\right),
$$


using translation

$$
\left(\begin{array}{l}
u \\
v
\end{array}\right)=T\left(\begin{array}{l}
u \\
V
\end{array}\right)
$$

system (4.2) becomes

$$
\left(\begin{array}{l}
U \\
V
\end{array}\right)=\left(\begin{array}{cc}
-1 & 0 \\
0 & \lambda_{2}
\end{array}\right)\left(\begin{array}{l}
U \\
V
\end{array}\right)+\left(\begin{array}{l}
f(U, V, \delta) \\
g(U, V, \delta)
\end{array}\right)
$$

where

$$
\begin{aligned}
& f(U, V, \delta) \\
& =\frac{a_{001}\left(\lambda_{2}-a_{100}\right)}{a_{010}\left(1+\lambda_{2}\right)} \delta+\frac{a_{200}\left(\lambda_{2}-a_{100}\right)-a_{010} b_{200}}{a_{010}\left(1+\lambda_{2}\right)} u^{2}+\frac{a_{110}\left(\lambda_{2}-a_{100}\right)-a_{010} b_{110}}{a_{010}\left(1+\lambda_{2}\right)} u v \\
& +\frac{a_{101}\left(\lambda_{2}-a_{100}\right)}{a_{010}\left(1+\lambda_{2}\right)} u \delta+\frac{a_{011}\left(\lambda_{2}-a_{100}\right)}{a_{010}\left(1+\lambda_{2}\right)} \nu \delta+\frac{a_{020}\left(\lambda_{2}-a_{100}\right)-a_{010} b_{020}}{a_{010}\left(1+\lambda_{2}\right)} v^{2} \\
& +\frac{a_{002}\left(\lambda_{2}-a_{100}\right)}{a_{010}\left(1+\lambda_{2}\right)} \delta^{2}+\frac{a_{300}\left(\lambda_{2}-a_{100}\right)-a_{010} b_{300}}{a_{010}\left(1+\lambda_{2}\right)} u^{3} \\
& +\frac{a_{210}\left(\lambda_{2}-a_{100}\right)-a_{010} b_{210}}{a_{010}\left(1+\lambda_{2}\right)} u^{2} v+\frac{a_{201}\left(\lambda_{2}-a_{100}\right)}{a_{010}\left(1+\lambda_{2}\right)} u^{2} \delta \\
& +\frac{a_{111}\left(\lambda_{2}-a_{100}\right)}{a_{010}\left(1+\lambda_{2}\right)} u v \delta+\frac{a_{102}\left(\lambda_{2}-a_{100}\right)}{a_{010}\left(1+\lambda_{2}\right)} u \delta^{2} \\
& +\frac{a_{120}\left(\lambda_{2}-a_{100}\right)-a_{010} b_{120}}{a_{010}\left(1+\lambda_{2}\right)} u v^{2}+\frac{a_{021}\left(\lambda_{2}-a_{100}\right)}{a_{010}\left(1+\lambda_{2}\right)} v^{2} \delta \\
& +\frac{a_{012}\left(\lambda_{2}-a_{100}\right)}{a_{010}\left(1+\lambda_{2}\right)} \nu \delta^{2}+\frac{a_{003}\left(\lambda_{2}-a_{100}\right)}{a_{010}\left(1+\lambda_{2}\right)} \delta^{3} \\
& +\frac{a_{030}\left(\lambda_{2}-a_{100}\right)-a_{010} b_{030}}{a_{010}\left(1+\lambda_{2}\right)} v^{3}+O\left((|u|+|v|+|\delta|)^{4}\right), \\
& =\frac{a_{001}\left(1+a_{100}\right)}{a_{010}\left(1+\lambda_{2}\right)} \delta+\frac{a_{200}\left(1+a_{100}\right)+a_{010} b_{200}}{a_{010}\left(1+\lambda_{2}\right)} u^{2}+\frac{a_{110}\left(1+a_{100}\right)+a_{010} b_{110}}{a_{010}\left(1+\lambda_{2}\right)} u v \\
& +\frac{a_{101}\left(1+a_{100}\right)}{a_{010}\left(1+\lambda_{2}\right)} u \delta+\frac{a_{011}\left(1+a_{100}\right)+a_{010} b_{011}}{a_{010}\left(1+\lambda_{2}\right)} v \delta+\frac{a_{020}\left(1+a_{100}\right)+a_{010} b_{020}}{a_{010}\left(1+\lambda_{2}\right)} v^{2} \\
& +\frac{a_{002}\left(1+a_{100}\right)}{a_{010}\left(1+\lambda_{2}\right)} \delta^{2}+\frac{a_{300}\left(1+a_{100}\right)+a_{010} b_{300}}{a_{010}\left(1+\lambda_{2}\right)} u^{3}+\frac{a_{210}\left(1+a_{100}\right)+a_{010} b_{210}}{a_{010}\left(1+\lambda_{2}\right)} u^{2} v \\
& +\frac{a_{201}\left(1+a_{100}\right)}{a_{010}\left(1+\lambda_{2}\right)} u^{2} \delta+\frac{a_{111}\left(1+a_{100}\right)}{a_{010}\left(1+\lambda_{2}\right)} u v \delta+\frac{a_{102}\left(1+a_{100}\right)}{a_{010}\left(1+\lambda_{2}\right)} u \delta^{2} \\
& +\frac{a_{120}\left(1+a_{100}\right)+a_{010} b_{120}}{a_{010}\left(1+\lambda_{2}\right)} u v^{2}+\frac{a_{021}\left(1+a_{100}\right)}{a_{010}\left(1+\lambda_{2}\right)} v^{2} \delta+\frac{a_{012}\left(1+a_{100}\right)}{a_{010}\left(1+\lambda_{2}\right)} v \delta^{2} \\
& +\frac{a_{003}\left(1+a_{100}\right)}{a_{010}\left(1+\lambda_{2}\right)} \delta^{3}+\frac{a_{030}\left(1+a_{100}\right)+a_{010} b_{030}}{a_{010}\left(1+\lambda_{2}\right)} v^{3}+O\left((|u|+|v|+|\delta|)^{4}\right), \\
& u=a_{010}(U+V), v=\left(-1-a_{100}\right) U+\left(\lambda_{2}-a_{100}\right) V .
\end{aligned}
$$


Now we determine the center manifold of $(4.3)$ at equilibrium point $(0,0)$ in a small neighborhood of $\delta=0$. We can obtain that there exists a center manifold by the center manifold theorem, which can be written as follows:

$$
W^{c}(0,0)=\left\{(U, V) \in R^{2}: Y=h(U, \delta)=c_{0} \delta+c_{1} U^{2}+c_{2} U \delta+c_{3} \delta^{2}+O\left((|U|+|\delta|)^{3}\right)\right\},
$$

where

$$
\begin{aligned}
c_{0}= & \frac{a_{001}\left(1+a_{100}\right)}{a_{010}\left(1-\lambda_{2}^{2}\right)}, \quad c_{1}=\frac{a_{200}\left(1+a_{100}\right)+a_{010} b_{200}}{a_{010}\left(1-\lambda_{2}^{2}\right)}, \\
c_{2}= & -\frac{c_{0}\left[a_{110}\left(1+a_{100}\right)+a_{010} b_{110}\right]+a_{101}\left(1+a_{100}\right)+2 c_{1} a_{001}\left(\lambda_{2}-a_{100}\right)}{a_{010}\left(1+\lambda_{2}\right)^{2}}, \\
c_{3}= & \frac{c_{0}\left[a_{011}\left(1+a_{100}\right)+a_{010} b_{011}\right]+c_{0}^{2}\left[a_{020}\left(1+a_{100}\right)+a_{010} b_{020}\right]+a_{002}\left(1+a_{100}\right)-c_{2} a_{001}\left(\lambda_{2}-a_{100}\right)}{a_{010}\left(1-\lambda_{2}^{2}\right)} \\
& -\frac{c_{1}\left[a_{001}\left(\lambda_{2}-a_{100}\right)\right]^{2}}{a_{010}^{2}\left(1+\lambda_{2}\right)\left(1-\lambda_{2}^{2}\right)} .
\end{aligned}
$$

We consider the following map originating from (4.3) restricted to the center manifold $W^{c}(0,0)$ :

$$
\begin{aligned}
F: U \rightarrow & -U+\frac{a_{001}\left(\lambda_{2}-a_{100}\right)}{a_{010}\left(1+\lambda_{2}\right)} \delta+\frac{a_{200}\left(\lambda_{2}-a_{100}\right)-a_{010} b_{200}}{a_{010}\left(1+\lambda_{2}\right)} u^{2} \\
& +\left[c_{0} \frac{a_{110}\left(\lambda_{2}-a_{100}\right)-a_{010} b_{110}}{a_{010}\left(1+\lambda_{2}\right)}+\frac{a_{101}\left(\lambda_{2}-a_{100}\right)}{a_{010}\left(1+\lambda_{2}\right)}\right] u \delta \\
& +\frac{a_{300}\left(\lambda_{2}-a_{100}\right)-a_{010} b_{300}}{a_{010}\left(1+\lambda_{2}\right)} u^{3} \\
& +\left[c_{0} \frac{a_{011}\left(\lambda_{2}-a_{100}\right)}{a_{010}\left(1+\lambda_{2}\right)}+c_{0}^{2} \frac{a_{020}\left(\lambda_{2}-a_{100}\right)-a_{010} b_{020}}{a_{010}\left(1+\lambda_{2}\right)}+\frac{a_{002}\left(\lambda_{2}-a_{100}\right)}{a_{010}\left(1+\lambda_{2}\right)}\right] \delta^{2} \\
+ & {\left[c_{0} \frac{a_{021}\left(\lambda_{2}-a_{100}\right)}{a_{010}\left(1+\lambda_{2}\right)}+c_{0} \frac{a_{012}\left(\lambda_{2}-a_{100}\right)}{a_{010}\left(1+\lambda_{2}\right)}+\frac{a_{003}\left(\lambda_{2}-a_{100}\right)}{a_{010}\left(1+\lambda_{2}\right)}\right.} \\
+ & \left.c_{0} \frac{a_{030}\left(\lambda_{2}-a_{100}\right)-a_{010} b_{030}}{a_{010}\left(1+\lambda_{2}\right)}\right] \delta^{3} \\
+ & {\left[c_{0} \frac{a_{111}\left(\lambda_{2}-a_{100}\right)}{a_{010}\left(1+\lambda_{2}\right)}+\frac{a_{102}\left(\lambda_{2}-a_{100}\right)}{a_{010}\left(1+\lambda_{2}\right)}+c_{0}^{2} \frac{a_{120}\left(\lambda_{2}-a_{100}\right)-a_{010} b_{120}}{a_{010}\left(1+\lambda_{2}\right)}\right] u \delta^{2} } \\
+ & {\left[c_{0} \frac{a_{210}\left(\lambda_{2}-a_{100}\right)-a_{010} b_{210}}{a_{010}\left(1+\lambda_{2}\right)}+\frac{a_{201}\left(\lambda_{2}-a_{100}\right)}{a_{010}\left(1+\lambda_{2}\right)}\right] u^{2} \delta+O(4) . }
\end{aligned}
$$

To enable Eq. (4.4) to undergo a flip bifurcation, it requires two discriminatory quantities $\eta_{1}$ and $\eta_{2}$ to be not zero, where

$$
\left\{\begin{array}{l}
\eta_{1}=\left(\frac{2 \partial^{2} F}{\partial U \partial \delta}+\frac{\partial F}{\partial \delta} \frac{\partial^{2} F}{\partial U^{2}}\right)_{(0,0)} \neq 0, \\
\eta_{2}=\left(\frac{1}{3} \frac{\partial^{3} F}{\partial U^{3}}+\frac{1}{2}\left(\frac{\partial^{2} F}{\partial U^{2}}\right)^{2}\right)_{(0,0)} \neq 0 .
\end{array}\right.
$$

Therefore, based on the above analysis and the theorem in [24], we obtain the following theorem. 
Theorem 4.1 If $\eta_{2} \neq 0$, the parameter $\delta$ alters in the limited region of the point $(0,0)$, then system (3.1) undergoes a flip bifurcation at $E_{*}$. Moreover, the period-2 orbit that bifurcates from $E_{*}$ is stable (unstable) if $\eta_{2}>0\left(\eta_{2}<0\right)$.

\subsection{N-S bifurcation}

From Theorem 3.1(4)(b), by using the bifurcation theorem [26-28] and selecting arbitrary parameters $\left(r_{2}, m, K, c, b, T, T_{n}, n\right)$, we write system (3.1) in the form

$$
\left\{\begin{array}{l}
x \rightarrow x \exp \left[\frac{r_{2}\left(1-\frac{x}{K}\right)(x-n)}{x+m}-\frac{b T x y}{1+c x+b T_{n} x^{2}}\right] \\
y \rightarrow x\left[1-\exp \left(-\frac{b T x y}{1+c x+b T_{n} x^{2}}\right)\right]
\end{array}\right.
$$

$E_{*}$ is the only positive equilibrium of system (4.5). We consider the following perturbation of (4.5), with $\delta_{*}$ used as the bifurcation parameter:

$$
\left\{\begin{array}{l}
x \rightarrow x \exp \left[\frac{\left(\delta_{*}+r_{2}\right)\left(1-\frac{x}{K}\right)(x-n)}{x+m}-\frac{b T x y}{1+c x+b T_{n} x^{2}}\right] \\
y \rightarrow x\left[1-\exp \left(-\frac{b T x y}{1+c x+b T_{n} x^{2}}\right)\right]
\end{array}\right.
$$

where $\left|\delta_{*}\right| \ll 1$.

Let $u=x-x_{*}, v=y-y_{*}$, the equilibrium $E_{*}$ is transformed to the origin point $(0,0)$, we obtain

$$
\left\{\begin{aligned}
u \rightarrow & a_{100} u+a_{010} v+a_{200} u^{2}+a_{110} u v+a_{020} v^{2}+a_{300} u^{3}+a_{210} u^{2} v \\
& +a_{120} u v^{2}+a_{030} v^{3}+O(4), \\
v \rightarrow & b_{100} u+b_{010} v+b_{200} u^{2}+b_{110} u v+b_{300} u^{3}+b_{210} u^{2} v+b_{020} v^{2} \\
& +b_{120} u v^{2}+b_{030} v^{3}+O(4),
\end{aligned}\right.
$$

where the coefficient is given in (4.2) and $r=\delta_{*}+r_{2}$. The characteristic equation associated with the linearization of system $(4.7)$ at $(0,0)$ is given by

$$
\lambda^{2}+p\left(\delta_{*}\right) \lambda+q\left(\delta_{*}\right)=0
$$

where

$$
\begin{aligned}
& p\left(\delta_{*}\right)=-\left[1+\left(\delta_{*}+r_{2}\right) x_{*} G-H+L\right], \\
& q\left(\delta_{*}\right)=\left(\delta_{*}+r_{2}\right) x_{*} G L+\frac{b T x_{*}^{2}}{M},
\end{aligned}
$$

we obtain

$$
\lambda_{1,2}=-\frac{p\left(\delta_{*}\right)}{2} \pm \frac{i}{2} \sqrt{4 q\left(\delta_{*}\right)-p^{2}\left(\delta_{*}\right)}
$$

and

$$
|\lambda|=\sqrt{q\left(\delta_{*}\right)}, \quad d=\left.\frac{d|\lambda|}{d \delta_{*}}\right|_{\delta_{*}=0}=\frac{x_{*} G L}{2} \neq 0 .
$$


Moreover, if $\delta_{*}=0$, we have $\lambda_{1,2}^{k} \neq 1(k=1,2,3,4)$, which is equivalent to $p(0) \neq-2,0,1,2$. Based on Theorem 3.1(b), we have $p(0) \neq-2,2$, then we only need to require $p(0) \neq 0,1$, which leads to

$$
(H-L) L,(H-L-1) L \neq 1-\frac{b T x^{2}}{M} .
$$

Therefore, the eigenvalues $\lambda_{1,2}$ do not lie in the intersection of the unit circle with the coordinate axes when $\delta_{*}=0$ and condition (4.9) holds.

Let $\delta_{*}=0, \mu=-\frac{p(0)}{2}, \omega=\frac{\sqrt{4 q(0)-p^{2}(0)}}{2}$, we make an invertible matrix:

$$
T=\left(\begin{array}{cc}
a_{010} & 0 \\
\mu-a_{100} & -\omega
\end{array}\right)
$$

using translation

$$
\left(\begin{array}{l}
u \\
v
\end{array}\right)=T\left(\begin{array}{l}
u \\
V
\end{array}\right)
$$

system (4.7) becomes

$$
\left(\begin{array}{l}
U \\
V
\end{array}\right)=\left(\begin{array}{cc}
\mu & -\omega \\
\omega & \mu
\end{array}\right)\left(\begin{array}{l}
U \\
V
\end{array}\right)+\left(\begin{array}{l}
\bar{f}(U, V) \\
\bar{g}(U, V)
\end{array}\right),
$$

where

$$
\begin{aligned}
\bar{f}(U, V)= & \frac{1}{a_{010}}\left(a_{200} u^{2}+a_{110} u v+a_{020} v^{2}+a_{300} u^{3}+a_{210} u^{2} v+a_{120} u v^{2}+a_{030} v^{3}\right)+O(4) \\
\bar{g}(U, V)= & \left(\frac{a_{200}\left(\mu-a_{100}\right)}{\omega a_{010}}-\frac{b_{200}}{\omega}\right) u^{2}+\left(\frac{a_{110}\left(\mu-a_{100}\right)}{\omega a_{010}}-\frac{b_{110}}{\omega}\right) u v \\
& +\left(\frac{a_{020}\left(\mu-a_{100}\right)}{\omega a_{010}}-\frac{b_{020}}{\omega}\right) v^{2} \\
& +\left(\frac{a_{300}\left(\mu-a_{100}\right)}{\omega a_{010}}-\frac{b_{300}}{\omega}\right) u^{3}+\left(\frac{a_{210}\left(\mu-a_{100}\right)}{\omega a_{010}}-\frac{b_{210}}{\omega}\right) u^{2} v \\
& +\left(\frac{a_{120}\left(\mu-a_{100}\right)}{\omega a_{010}}-\frac{b_{120}}{\omega}\right) u v^{2} \\
& +\left(\frac{a_{030}\left(\mu-a_{100}\right)}{\omega a_{010}}-\frac{b_{030}}{\omega}\right) v^{3}+O\left((|u|+|v|)^{4}\right)
\end{aligned}
$$

and

$$
\begin{aligned}
& u^{2}=a_{010}^{2} U^{2}, \quad u v=a_{010}\left(\mu-a_{100}\right) U^{2}-a_{010} \omega U V, \\
& v^{2}=\left(\mu-a_{100}\right)^{2} U^{2}-2 \omega\left(\mu-a_{100}\right) U V+\omega^{2} V^{2}, \quad u^{3}=a_{010}^{3} U^{3}, \\
& u^{2} v=a_{010}^{2}\left(\mu-a_{100}\right) U^{3}-a_{010}^{2} \omega U^{2} V, \\
& u v^{2}=a_{010}\left(\mu-a_{100}\right)^{2} U^{3}-2 a_{010} \omega U^{2} V+a_{010} \omega^{2} U V^{2}, \\
& v^{3}=\left(\mu-a_{100}\right) U^{3}-\omega^{3} V^{3}-3 \omega\left(\mu-a_{100}\right)^{2} U^{2} V+3\left(\mu-a_{100}\right) \omega^{2} U V^{2} .
\end{aligned}
$$


Therefore

$$
\begin{aligned}
& \bar{f}_{U U}=2 a_{200} a_{010}+2 a_{110}\left(\mu-a_{100}\right)+\frac{2 a_{020}\left(\mu-a_{100}\right)^{2}}{a_{010}}, \\
& \bar{f}_{U V}=-a_{110} \omega-\frac{2 a_{020} \omega\left(\mu-a_{100}\right)}{a_{010}}, \quad \bar{f}_{V V}=\frac{2 a_{020} \omega^{2}}{a_{010}}, \\
& \bar{f}_{\text {UUU }}=6 a_{300} a_{010}^{2}+6 a_{210} a_{010}\left(\mu-a_{100}\right)+6 a_{120}\left(\mu-a_{100}\right)^{2}+\frac{6 a_{030}\left(\mu-a_{100}\right)}{a_{010}}, \\
& \bar{f}_{U U V}=-2 a_{010} a_{210} \omega-4 a_{120} \omega-\frac{6 a_{030} \omega\left(\mu-a_{100}\right)^{2}}{a_{010}}, \quad \bar{f}_{V V V}=-\frac{6 a_{030} \omega^{3}}{a_{010}}, \\
& \bar{f}_{U V V}=2 a_{120} \omega^{2}+\frac{6 a_{030}\left(\mu-a_{100}\right) \omega^{2}}{a_{010}}, \\
& \bar{g}_{U U}=2 a_{010}^{2}\left(\frac{a_{200}\left(\mu-a_{100}\right)}{\omega a_{010}}-\frac{b_{200}}{\omega}\right)+2 a_{010}\left(\mu-a_{100}\right)\left(\frac{a_{110}\left(\mu-a_{100}\right)}{\omega a_{010}}-\frac{b_{110}}{\omega}\right) \\
& +2\left(\mu-a_{100}\right)^{2}\left(\frac{a_{020}\left(\mu-a_{100}\right)}{\omega a_{010}}-\frac{b_{020}}{\omega}\right) \text {, } \\
& \bar{g}_{U V}=-a_{010} \omega\left(\frac{a_{110}\left(\mu-a_{100}\right)}{\omega a_{010}}-\frac{b_{110}}{\omega}\right)-2 \omega\left(\mu-a_{100}\right)\left(\frac{a_{020}\left(\mu-a_{100}\right)}{\omega a_{010}}-\frac{b_{020}}{\omega}\right), \\
& \bar{g}_{V V}=2 \omega^{2}\left(\frac{a_{020}\left(\mu-a_{100}\right)}{\omega a_{010}}-\frac{b_{020}}{\omega}\right) \text {, } \\
& \bar{g}_{\text {UUU }}=6 a_{010}^{3}\left(\frac{a_{300}\left(\mu-a_{100}\right)}{\omega a_{010}}-\frac{b_{300}}{\omega}\right)+6 a_{010}^{2}\left(\mu-a_{100}\right)\left(\frac{a_{210}\left(\mu-a_{100}\right)}{\omega a_{010}}-\frac{b_{210}}{\omega}\right) \\
& +6 a_{010}\left(\mu-a_{100}\right)^{2}\left(\frac{a_{120}\left(\mu-a_{100}\right)}{\omega a_{010}}-\frac{b_{120}}{\omega}\right) \\
& +6\left(\mu-a_{100}\right)\left(\frac{a_{030}\left(\mu-a_{100}\right)}{\omega a_{010}}-\frac{b_{030}}{\omega}\right) \text {, } \\
& \bar{g}_{U V V}=2 a_{010} \omega^{2}\left(\frac{a_{120}\left(\mu-a_{100}\right)}{\omega a_{010}}-\frac{b_{120}}{\omega}\right)+6\left(\mu-a_{100}\right) \omega^{2}\left(\frac{a_{030}\left(\mu-a_{100}\right)}{\omega a_{010}}-\frac{b_{030}}{\omega}\right), \\
& \bar{g}_{U U V}=-2 a_{010}^{2} \omega\left(\frac{a_{210}\left(\mu-a_{100}\right)}{\omega a_{010}}-\frac{b_{210}}{\omega}\right)-4 a_{010} \omega\left(\frac{a_{120}\left(\mu-a_{100}\right)}{\omega a_{010}}-\frac{b_{120}}{\omega}\right) \\
& -6 \omega\left(\mu-a_{100}\right)^{2}\left(\frac{a_{030}\left(\mu-a_{100}\right)}{\omega a_{010}}-\frac{b_{030}}{\omega}\right) \text {, } \\
& \bar{g}_{V V V}=-6 \omega^{3}\left(\frac{a_{030}\left(\mu-a_{100}\right)}{\omega a_{010}}-\frac{b_{030}}{\omega}\right) .
\end{aligned}
$$

To enable system (4.7) to undergo an N-S bifurcation, we require the following discriminatory quantity $\theta$ to be not zero:

$$
\theta=-\left[\operatorname{Re}\left(\frac{(1-2 \lambda) \bar{\lambda}^{2}}{1-\lambda} \xi_{20} \xi_{11}\right)-\frac{1}{2}\left|\xi_{11}\right|^{2}-\left|\xi_{02}\right|^{2}+\operatorname{Re}\left(\bar{\lambda} \xi_{21}\right)\right]_{\delta=0},
$$

where

$$
\xi_{20}=\frac{1}{8}\left[\bar{f}_{U U}-\bar{f}_{V V}+2 \bar{g}_{U V}+i\left(\bar{g}_{U U}-\bar{g}_{V V}-2 \bar{f}_{U V}\right)\right],
$$




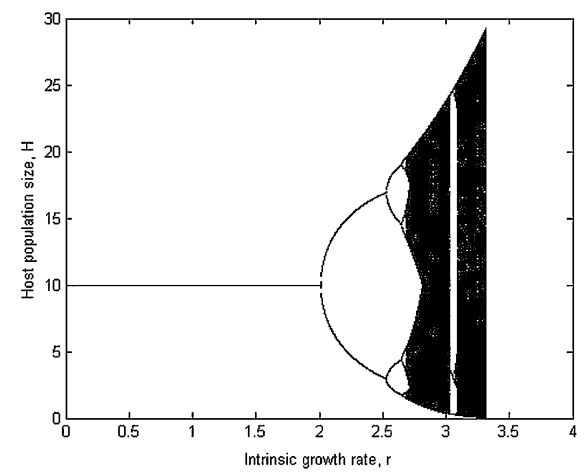

(a)

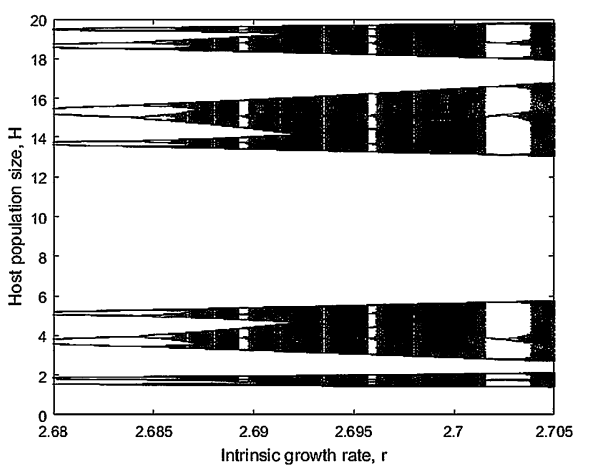

(b)

Figure 1 Numerical simulations of equation (3.1) (a) Flip bifurcation of system (3.1) for $T=1, T_{n}=0.4, b=0.05$, $c=0.2, m=0.01, n=0.05, K=10$, and $1.5 \leq r \leq 3$, (b) NS-bifurcation of system (1.2) for $2.68 \leq r \leq 2.705$

$$
\begin{aligned}
& \xi_{11}=\frac{1}{4}\left[\bar{f}_{U U}+\bar{f}_{V V}+i\left(\bar{g}_{U U}+\bar{g}_{V V}\right)\right], \\
& \xi_{02}=\frac{1}{8}\left[\bar{f}_{U U}-\bar{f}_{V V}-2 \bar{g}_{U V}+i\left(\bar{g}_{U U}-\bar{g}_{V V}+\bar{f}_{U V}\right)\right], \\
& \xi_{21}=\frac{1}{16}\left[\bar{f}_{U U U}+\bar{f}_{V V V}+2 \bar{g}_{U U V}+i\left(\bar{g}_{U U U}+\bar{g}_{U V V}-2 \bar{f}_{V V V}\right)\right] .
\end{aligned}
$$

Therefore, according to the above analysis and the theorem in [24], we obtain the following theorem.

Theorem 4.2 System (3.1) undergoes an $N$-S bifurcation at equilibrium $E_{*}$ if conditions in Theorem 3.1(4)(b) and $\theta \neq 0$ hold and $\delta_{*}$ varies in a small vicinity of the origin. Moreover, if $\theta<0$ (or $\theta>0)$, then an attracting (or repelling) invariant closed curve bifurcates from $E_{*}$ for $\delta_{*}>0\left(\right.$ or $\left.\delta_{*}<0\right)$.

\section{Numerical simulation}

In this section, we present the interesting and complex dynamic behavior of discrete systems by numerical simulation.

Figure 1 is a numerical simulation of system (3.1), and we set $T=1, T_{n}=0.4, b=0.05$, $c=0.2, m=0.01, n=0.05, K=10$, the initial number of host and parasite populations $\left(x_{0}, y_{0}\right)=(5,2.5)$, and the number of host populations and parasite populations changes as $r$ increases. The bifurcation parameters are considered in the following two cases.

Case 1: It can be observed from Fig. 1(a) that when $r<2.014$, the equilibrium point is stable, when $r>2.014$, it loses its stability, from one cycle to two cycles, and produces a flip bifurcation. As $r$ continues to increase, periodic oscillations are observed with periods $4, \ldots$, which eventually leads to chaos.

Case 2: Let the parameter $r$ vary in the range $2.68 \leq r \leq 2.705$, we can see according to Fig. 1(b) that the N-S bifurcation occurs when $r=2.684$, and an attracting invariant closed curve appears if $r>2.684$.

Figure 2 shows a fascinating and complex dynamical structure including bifurcation phenomena previously encountered in Fig. 1(a) and (b) with $T=5, T_{n}=0.4, b=0.05$, $c=0.2, m=0.01, n=0.05, K=10$ (namely period-doubling cascades, chaotic bands, and attractor crisis [20]). 


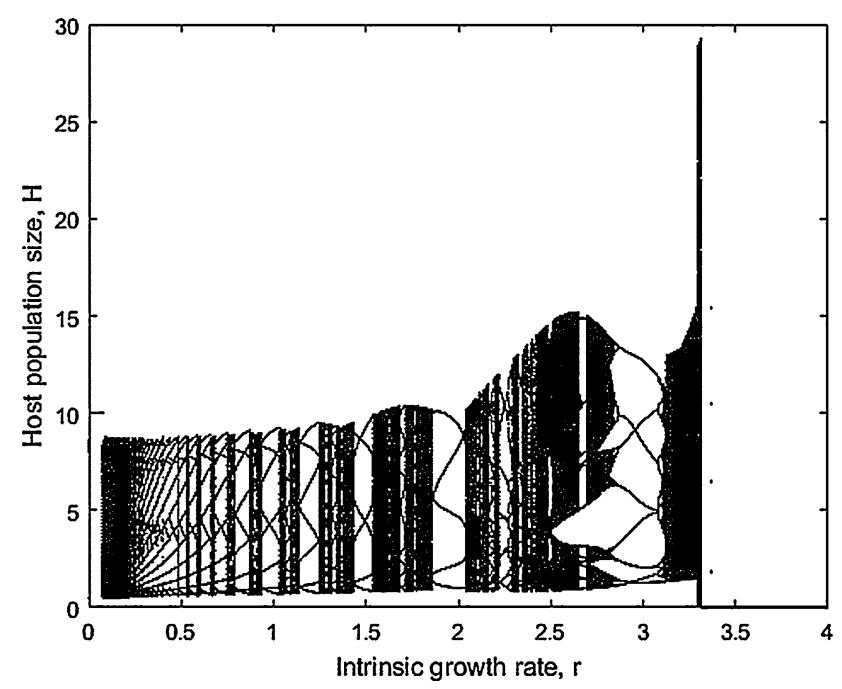

Figure 2 Bifurcation diagram of the host population in the Holling type III host-parasitoid model (3.1) for $0 \leq r \leq 4, T=5$

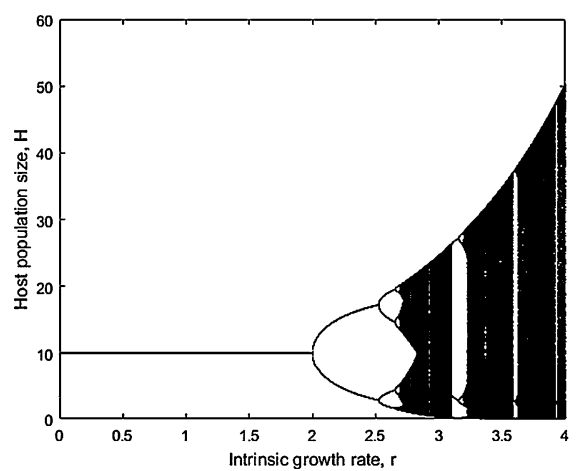

(a)

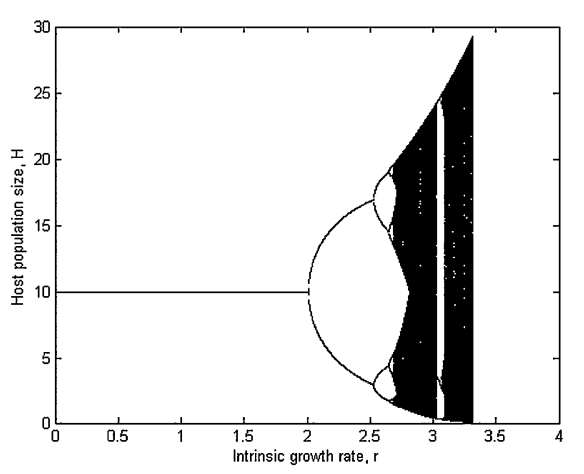

(b)

Figure 3 Numerical simulations of equation (3.1). (a) System (3.1) without Allee effect for varying $r$ in range $0 \leq r \leq 4$ and $K=10, T=1, T_{n}=0.4, b=0.05, c=0.2, m=0, n=0$; (b) System (3.1) with Allee effect. Initial value as $\left(x_{0}, y_{0}\right)=(5,2.5)$, and $m=0.01, n=0.05$

To better understand the impact of the Allee effect, we simulate system (3.1) with and without Allee effect (Fig. 3 and Fig. 4). From Fig. 3, when the parameter $r<3.32$, the dynamics of the population with Allee effect are roughly the same as those without Allee effect. When there is no Allee effect and $r>3.32$, the population of host survives, even when $r=4$, the population of host still exists; however, the maximum value of host populations with Allee effect grows to 29 and becomes extinct at $r=3.32$. Therefore, we conclude that the Allee effect is a factor affecting the dynamic change of the system. The extinction of population will be accelerated by the Allee effect, and the whole system will collapse. Figure 4 shows the basins of attraction with $r=3, K=5, T=100, T_{n}=1, c=0.03, b=0.008$ except $x_{0}$ and $y_{0}$, the influence of the Allee effect on the dynamic complexity of a hostparasitic system is given from example. Comparing Fig. 4(a) with Fig. 4(b), we suppose 


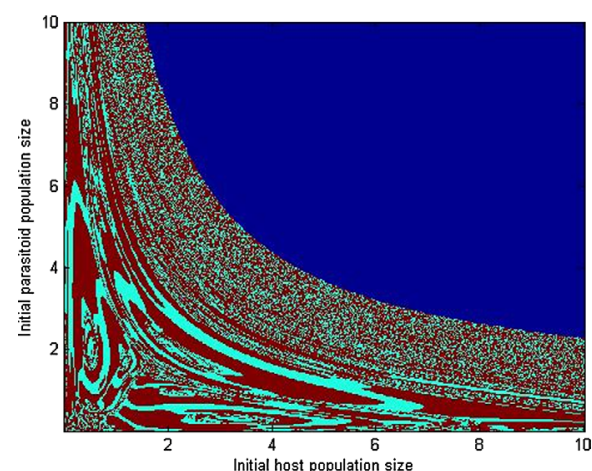

(a)

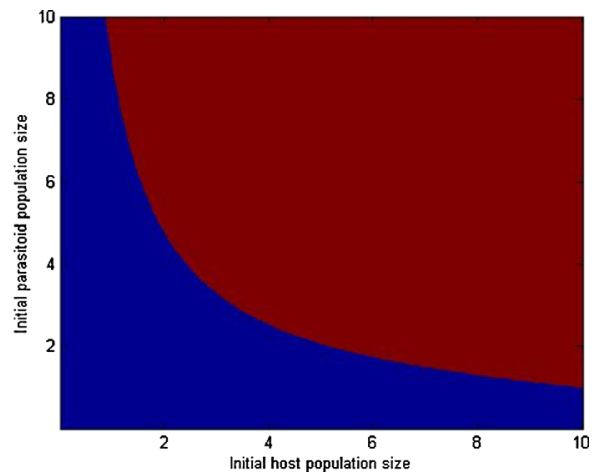

(b)

Figure 4 The basins of attraction for non-unique attractors, the scopes of initial values $\left(x_{0}\right.$ and $\left.y_{0}\right):(0,10]$. (a) Host-parasitoid system without Allee effect for the host $(m=0, n=0)$ : the blue, sky blue, and brown areas are the basins of attraction for chaotic, period-3, and period-6; (b) host-parasitoid system incorporating Allee effect $(m=0.01, n=0.01)$ : the blue area: the basins of chaotic; brown area:the basins of period-1

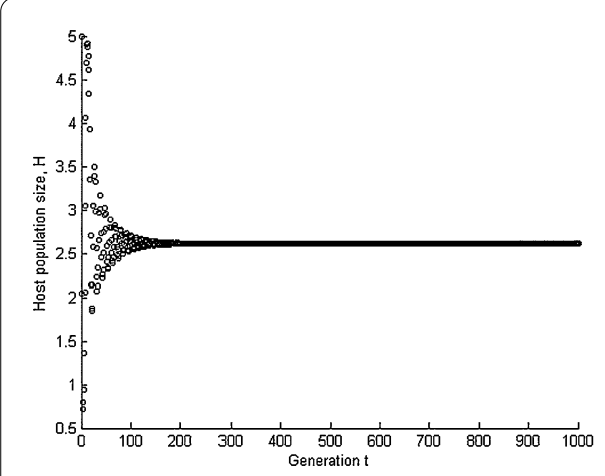

(a)

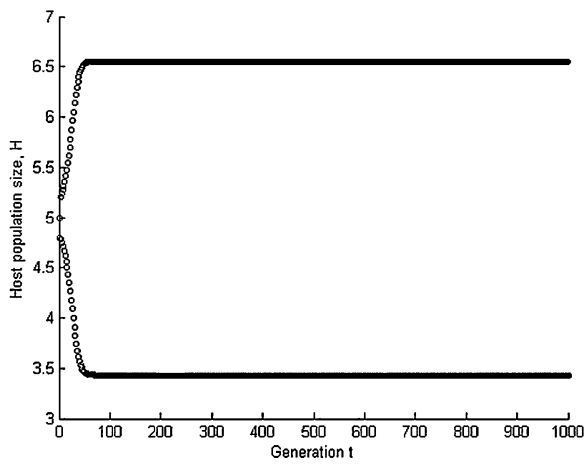

(b)

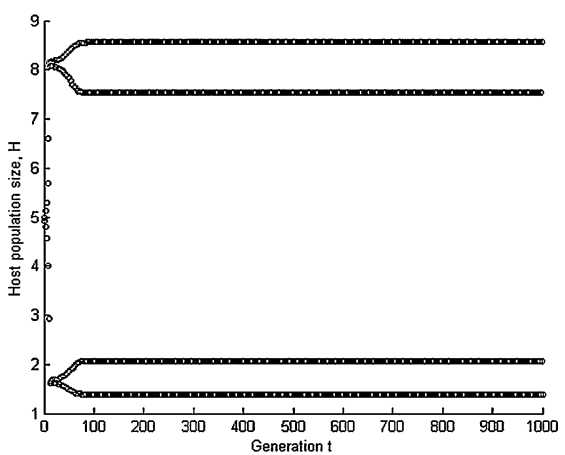

(c)

Figure 5 Changes of the host population size over time. We fix $T=1, T_{n}=0.4, K=5$, and: (a) $r=1, b=0.5$, $c=0.2, m=1, n=0.005 ;$ (b) $m=0.01, n=0.05, r=2.1, c=0.2, b=0.00697 ;(\mathbf{c}) n=0.05, r=2.6, c=0.8$, $b=0.00697, m=0.099$

that the sensitivity of population dynamics to the initial conditions after addition of the Allee effect is reduced. In sum, the Allee effect plays a crucial role in stabilizing a hostparasitoid system. 


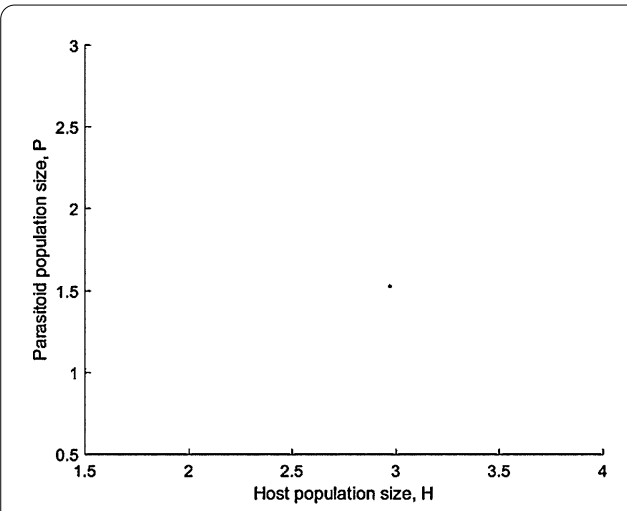

(a)

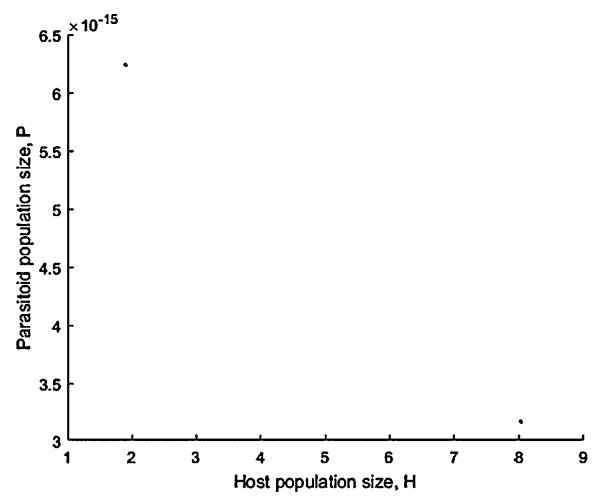

(b)

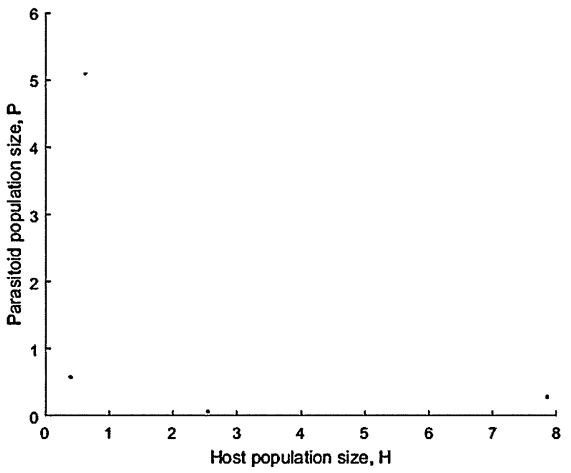

(c)

Figure 6 Three alternative attractors (1-cycle, 2-cycle, 4-cycle) for $K=5, n=0.05, m=0.01, c=0.03, b=0.08$, and (a) $T=23, T_{n}=1, r=1.81$; (b) $T=23, T_{n}=10 ; r=2.4$; (c) $T=100, T_{n}=1, r=2.6$

Moreover, it appears that the attractor is non-unique [20]: in this case the alternative attractors are, for example, period-one, period-two, and period-four attractors (Fig. 5 and Fig. 6).

\section{Conclusions}

In population study, except for the existing focus on the dynamic characteristics and structure of population, we should pay more attention to the evolutionary law of the interaction between populations. The Allee effect, an ecological phenomenon, has potential influence on population dynamics [29]. In this paper we establish a type of Holling type III functional response discrete host-parasite system with Allee effect. Firstly, we analyze the persistence of this system and obtain the conditions in which the system will be persistent. Then we analyze the stability of four equilibrium points of the system, obtain the conditions of local stability, and prove that with certain parameters the system allows for bifurcation. Thirdly, the system is numerically simulated, and it can be observed that when the host's intrinsic growth rate differs, the system will undergo both a flip bifurcation and a Neimark-Sacker bifurcation. Finally, the comparative analysis of population affected by both presence and absence of the Allee effect is given. According to the figures, we find that the Allee effect not only reduces the complexity of population dynamics, but also accelerates the extinction of a system. Therefore, we suppose that the Allee effect can condition the dynamic changes of a system. 
We also know from numerical simulation that the behavior of the system after a long time depends not only on the initial state, but also on the size of parameters. Slight changes in the parameter values and initial values may greatly influence the dynamic behavior of population [30-34].

\section{Funding}

This work was supported by the Fundamental Research Funds for the Central Universities (31920180116, 31920180044, 31920170072), the National Natural Science Foundation of China (31260098, 31560127), the Program for Young Talent of State Ethnic Affairs Commission of China (No. [2014]121), the Central Universities Fundamental Research Funds for the Graduate Students of Northwest Minzu University (Yxm2019109), and Gansu Provincial First-Class Discipline Program of Northwest Minzu University (No. 11080305).

\section{Availability of data and materials}

Not applicable.

\section{Competing interests}

The authors declare that they have no competing interests.

\section{Authors' contributions}

All authors contributed equally and significantly in this paper. All authors read and approved the final manuscript.

\section{Author details}

1 School of Mathematics and Computer Science, Northwest Minzu University, Lanzhou, P.R. China. ${ }^{2}$ Experimental Teaching Department, Northwest Minzu University, Lanzhou, P.R. China.

\section{Publisher's Note}

Springer Nature remains neutral with regard to jurisdictional claims in published maps and institutional affiliations.

Received: 11 July 2019 Accepted: 25 November 2019 Published online: 11 December 2019

\section{References}

1. Hastings, A., Powell, T.: Chaos in three-species food chain. Ecology 72, 896-903 (1991)

2. Liu, X.J., Chu, Y.D., Liu, Y.: Bifurcation and chaos in a host-parasitoid system with a lower bound for the host. Adv. Differ. Equ. 2018, $31(2018)$

3. Kang, Y., Sasmal, S.K., Bhowmick, A.R., Chattopadhyay, J.: A host-parasitoid system with predation-driven component Allee effects in host population. J. Biol. Dyn. 9, 213-232 (2015)

4. Cheng, L., Cao, H.: Bifurcation analysis of a discrete-time ratio-dependent predator-prey system with Allee effect. Commun. Nonlinear Sci. Numer. Simul. 38, 288-302 (2016)

5. Jana, D., Elsayed, E.M.: Interplay between strong Allee effect, harvesting and hydra effect of a single population discrete-time system. Int. J. Biomath. 9, 1650004 (2016)

6. Rana, S., Bhowmick, A.R., Bhattacharya, S.: Impact of prey refuge on a discrete time predator-prey system with Allee effect. Int. J. Bifurc. Chaos 24, 1450106 (2014)

7. Liu, H., Li, Z.Z., Gao, M., Dai, H.W., Liu, Z.G.: Dynamics of a host-parasitoid system with Allee effect for the host and parasitoid aggregation. Ecol. Complex. 6, 337-345 (2009)

8. Liu, H., Ye, Y., Wei, Y.M., Ma, M., Ye, J.H.: Dynamic study of a predator-prey system with weak Allee effect and Holling type-III functional response. Dyn. Syst. Appl. 27(4), 943-953 (2018)

9. Sasmal, S.K., Chattopadhyay, J.: An eco-epidemiological system with infected prey and predator subject to the weak Allee effect. Math. Biosci. 246, 260-271 (2013)

10. Sasmal, S.K., Bhowmick, A.R., Al-Khaled, K., Bhattacharya, S., Chattopadhyay, J.: Interplay of functional responses and weak Allee effect on pest control via viral infection or natural predator: an eco-epidemiological study. Differ. Equ. Dyn. Syst. 24, 21-50 (2016)

11. Ye, Y., Liu, H., Wei, Y.M., Zhang, K., Ma, M.: Dynamic study of a predator-prey model with Allee effect and Holling typefunctional response. Adv. Differ. Equ. 2019, Article ID 369 (2019)

12. Ye, Y., Liu, H., Wei, Y.M., Ma, M., Zhang, K.: Dynamic study of a predator-prey model with weak Allee effect and delay. Adv. Math. Phys., 2019, Article ID 7296461 (2019)

13. Tian, B., Yang, L., Zhong, S.: Global stability of a stochastic predator-prey system with Allee effect. Int. J. Biomath. 8, 1550044 (2015)

14. Shi, L., Liu, H., Wei, Y.M., Ma, M., Ye, J.H.: The permanence and periodic solution of a competitive system with infinite delay, feedback control, and Allee effect. Adv. Differ. Equ. 2018, 10 (2018)

15. LV, S., Zhao, M.: The dynamic complexity of a host-parasitoid system with a lower bound for the host. Chaos Solitons Fractals 36, 911-919(2008)

16. Veijo, K., Janica, Y., Mikko, H.: Dynamic complexities in host-parasitoid interaction. J. Theor. Biol. 197, $331-341$ (1999)

17. Tang, S.Y., Chen, L.S.: Chaos in functional response host-parasitoid ecosystem systems. Chaos Solitons Fractals 13 , 875-884 (2002)

18. May, R.M.: Simple mathematical systems with very complicated dynamics. Nature $261,459-467$ (1976)

19. Oaten, A., Murdoch, W.W.: Functional response and stability in predator-prey system. Am. Nat. 109(967), 299-318 (1975)

20. Murdoch, W.W., Oaten, A.: Predation and population stability. Adv. Ecol. Res. 9, 1-131 (1975) 
21. Chen, F: Some new results on the permanence and extinction of nonautonomous Gilpin-Ayala type competition system with delays. Nonlinear Anal., Real World Appl. 7(5), 1205-1222 (2006)

22. Chen, F.D.: Permanence for the discrete mutualism system with time delays. Math. Comput. Model. 47, 431-435 (2008)

23. Carr, J.: Application of Center Manifold Theory. Springer, New York (1981)

24. Guckenheimer, J., Holmes, P.: Nonlinear Oscillations, Dynamical Systems, and Bifurcations of Vector Fields. Springer, New York (1983)

25. Jang, S.R., Diamond, S.L.: A host-parasitoid interaction with Allee effects on the host. Comput. Math. Appl. 53, 89-103 (2007)

26. Kuznetsov, Y.A.: Elements of Applied Bifurcation Theory, 2nd edn. Springer, New York (1998)

27. Sohel Rana, S.M., Kulsum, U.: Bifurcation analysis and chaos control in a discrete-time predator-prey system of Leslie type with simplified Holling type IV functional response. Discrete Dyn. Nat. Soc. 2017, Article ID 9705985 (2017)

28. Liu, F., Yin, X., Sun, F., Wang, H.Q.: Bifurcation analysis and chaotic behavior of a discrete-time delayed genetic oscillator system. Adv. Differ. Equ. 2017, 3 (2017)

29. Banerjee, M., Takeuchi, Y: Maturation delay for the predators can enhance stable coexistence for a class of prey-predator systems. J. Theor. Biol. 412, 154-171 (2017)

30. Xu, C.L., Mark, S.B.: Dynamic complexities in a mutual interference host-parasitoid system. Chaos Solitons Fractals 24, 175-182 (2005)

31. Huang, S., Tian, Q: Marcinkiewicz estimates for solution to fractional elliptic Laplacian equation. Comput. Math. Appl. 78, 1732-1738 (2019)

32. Liu, Z.G., Zhang, F.P., Hui, C.: Density dependent dispersal complicates spatial synchrony in tri-trophic food chains. Popul. Ecol. 58(1), 223-230 (2016)

33. Liu, Z.G., Zhang, F.P.: Species coexistence of communities with intraguild predation: the role of refuges used by the resource and the intraguild prey. Biosystems 114, 25-30 (2013)

34. Liu, Z.G., Gao, M., Zhang, F.P., Li, Z.Z.: Synchrony of Spatial Populations Induced by Colored Environmental Noise and Dispersal. Biosystems 98(2), 115-121 (2009)

\section{Submit your manuscript to a SpringerOpen ${ }^{\circ}$ journal and benefit from:}

- Convenient online submission

- Rigorous peer review

- Open access: articles freely available online

- High visibility within the field

- Retaining the copyright to your article

Submit your next manuscript at $\gg$ springeropen.com 